\title{
Fundamental piezo-Hall coefficients of single crystal p-type 3C-SiC for arbitrary crystallographic orientation
}

\author{
Afzaal Qamar, ${ }^{1, \text { a) }}$ Dzung Viet Dao, ${ }^{1,2}$ Hoang-Phuong Phan, ${ }^{1}$ Toan Dinh, ${ }^{1}$ and Sima Dimitrijev ${ }^{1,2}$ \\ 1) Queensland Micro- and Nanotechnology Centre, Griffith University, Queensland, 4111, \\ Australia \\ 2) School of Engineering, Griffith University, Queensland, 4222, Australia
}

Piezo-Hall effect in single crystal p-type 3C-SiC, grown by LPCVD process has been characterized for various crystallographic orientations. The quantified values of the piezo-Hall effect in heavily doped p-type $3 \mathrm{C}$ $\mathrm{SiC}(100)$ and 3C-SiC(111) for different crystallographic orientations were used to obtain the fundamental piezo-Hall coefficients, $P_{12}=(5.3 \pm 0.4) \times 10^{-11} \mathrm{~Pa}^{-1}, P_{11}=(-2.6 \pm 0.6) \times 10^{-11} \mathrm{~Pa}^{-1}$ and $P_{44}=(11.42 \pm 0.6) \times 10^{-11}$ $\mathrm{Pa}^{-1}$. Unlike the piezoresistive effect, the piezo-Hall effect for (100) and (111) planes is found to be independent of the angle of rotation of the device within the crystal plane. The values of fundamental piezo-Hall coefficients obtained in this study can be used to predict the piezo-Hall coefficients in any crystal orientation which is very important for designing of 3C-SiC Hall sensors to minimize the piezo-Hall effect for stable magnetic field sensitivity.

Hall-effect devices have been used for applications in different areas of applied physics due to there ability to sense the magnetic field. The magnetic field sensitivity of these devices can be affected significantly by the stresses introduced into the device due to various factors which include several fabrication and post fabrication issues ${ }^{1-5}$. These issues are high growth temperatures, a thermal mismatch between mounting material and chip in die attachment, mechanical impact during device operation and the most important is the encapsulation of fabricated device. Due to the introduction of stress into the device by these factors, the design parameters, temperature behavior and offset voltage of the device will be altered leading to the variation in magnetic field sensitivity of the device ${ }^{1}$. The piezo-Hall effect has been studied for $\mathrm{Si}$ based Hall devices for years and a large number of studies on Si can be found in literature ${ }^{6-9}$. The piezo-Hall coefficients for n-type Si which can quantify this effect in any crystal orientation were also determined and have been used to compensate for the stress related magnetic field drifts of Si based Hall devices in various studies ${ }^{1-9}$. The need of Hall devices to operate in harsh environment of high temperature, high radiation, high frequency and high power limits the use of Si based Hall devices and alternate materials have to be considered for these applications including, SiC, GaAs and GaN.

The capability of hetero-epitaxial growth of $\mathrm{SiC}$ combined with its high thermal conductivity, low thermal-expansion coefficient, better mechanical strength, and chemical stability make it a promising material for harsh environment applications ${ }^{10,11}$. SiC can be found in various polytypes, including $4 \mathrm{H}-\mathrm{SiC}, 6 \mathrm{H}-\mathrm{SiC}$, and $3 \mathrm{C}-\mathrm{SiC}$. $3 \mathrm{C}-\mathrm{SiC}$ is compatible with the existing process of micro-electro-mechanical systems (MEMS) and it can be grown over Si substrates of different crystal orientations, e.g. (100), (110) or $(111)^{12,13}$. 3C-SiC has been investigated for various piezo-effects recently, in-

\footnotetext{
a)Electronic mail: afzaal.qamar@griffithuni.edu.au.
}

cluding piezoresistive, piezoelectric, piezo-junction and pseudo-Hall effects and it has been found that the $3 \mathrm{C}$ $\mathrm{SiC}$ is a promising material for strain or stress sensing applications ${ }^{14-21}$. Phan et. al. ${ }^{15}$ reported the fundamental piezoresistive coefficients of p-type 3C-SiC.

The piezo-Hall effect with piezo-Hall coefficient $P_{11}$ was reported recently in ${ }^{22}$ using a rectangular Hall device with only one direction of stress and current. But the measurement of complete set of piezo-Hall coefficients $P_{11}, P_{12}$ and $P_{44}$ which is still missing, requires different crystal growth direction along with different directions of applied stress and current to check their dependence on crystallographic orientation. Therefore, this paper aims to investigate the full set of piezo-Hall coefficients of single crystal p-type 3C-SiC and their dependence on crystal orientation using the Hall devices grown by LPCVD in (100) and (111) crystallographic planes. Additionally, the Greek cross shape which is the standard and much stable shape for Hall devices is used in this study. The results achieved in this study can be used to explain the stress related drifts in magnetic field sensitivity of p-type single crystal 3C-SiC Hall devices.

Single crystal p-type 3C-SiC(100) and 3C-SiC(111) thin films were grown to a thickness of 300nm on $\mathrm{Si}(100)$ and $\mathrm{Si}(111)$ substrates by LPCVD process at low temperature $\left(1000^{\circ} \mathrm{C}\right)$. Alternating supply epitaxy (ASE) was employed to grow the single crystal 3C-SiC(100)/Si(100) and $3 \mathrm{C}-\mathrm{SiC}(111) / \mathrm{Si}(111)$ and the precursors $\mathrm{SiH}_{4}$ and $\mathrm{C}_{3} \mathrm{H}_{6}$ were employed as source of $\mathrm{Si}$ and $\mathrm{C}$ atoms. In situ doping of the films was performed with Trimethyaluminium (TMAl) as a source of $\mathrm{Al}$ (p-type dopant) ${ }^{23,24}$. After the growth process, X-ray diffraction (XRD) analysis of the grown films was carried out to confirm the crystal structure followed by the rocking curve to analyze the crystalline quality. Figure 1 (a) shows the XRD pattern of $3 \mathrm{C}-\mathrm{SiC}(100)$ thin film on $\mathrm{Si}(100)$ in conventional $\theta-2 \theta$ scan mode. It can be confirmed from Fig. 1(a) that only the peaks corresponding to (100) plane are present which confirms that single crystal $3 \mathrm{C}-\mathrm{SiC}(100)$ was grown on $\mathrm{Si}(100)$. The inset of Fig. 1(a) shows the rocking curve 
This manuscript was accepted by Appl. Phys. Lett. Click here to see the version of record.

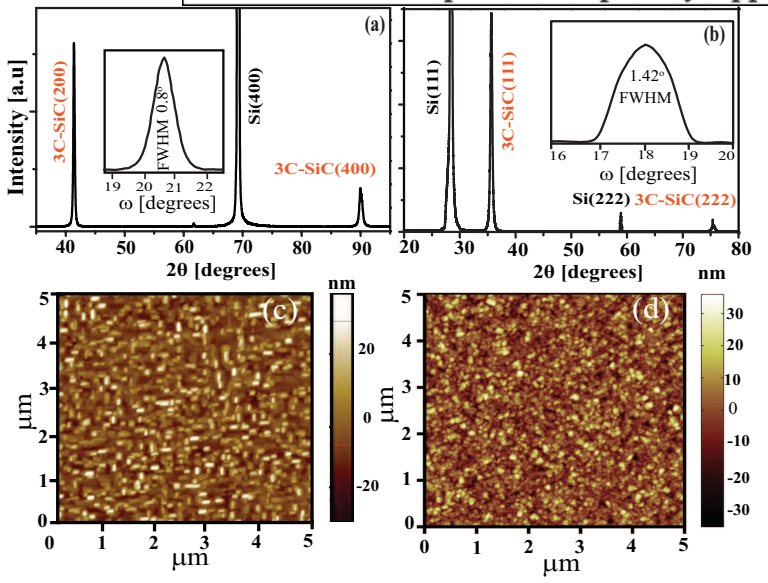

FIG. 1. (a) XRD pattern of $3 \mathrm{C}-\mathrm{SiC}(100)$, the inset shows the rocking curve, (b) XRD pattern of 3C-SiC(111) with rocking curve shown in inset, (c) AFM image of $3 \mathrm{C}-\mathrm{SiC}(100)$, (d) AFM image of 3C-SiC(111).

of $3 \mathrm{C}-\mathrm{SiC}(100)$ peak and the observed full width at half maximum (FWHM) value is $0.80^{\circ}$ which shows the good crystalline quality of the grown film. Figure 1(b) shows the $\theta-2 \theta$ scan of $3 \mathrm{C}-\mathrm{SiC}(111)$ grown on $\mathrm{Si}(111)$ and the rocking curve is shown in inset of Fig. 1(b) which confirms that the grown film is single crystalline and the observed value of FWHM $\left(1.42^{\circ}\right)$ is also reasonably good. Atomic force microscopy (AFM) was used to measure the roughness of the grown thin films. The AFM images of $3 \mathrm{C}-\mathrm{SiC}(100)$ and $3 \mathrm{C}-\mathrm{SiC}(111)$ thin films for a scan area of $5 \mu \mathrm{m} \times 5 \mu \mathrm{m}$ are shown in Fig. 1(c) and Fig. 1(d), respectively. 3C-SiC(100) has root mean square (RMS) roughness of $20 \pm 0.5 \mathrm{~nm}$ while RMS roughness of $3 \mathrm{C}-\mathrm{SiC}(111)$ was found to be $8.6 \pm 0.5 \mathrm{~nm}$. Electrical properties of the grown film were characterized using Hall effect measurements. The carrier concentration of p-type single crystalline 3C-SiC(100) and 3C-SiC(111) was found to be $5 \times 10^{18} \mathrm{~cm}^{-3}$ and $8 \times 10^{18} \mathrm{~cm}^{-3}$, respectively. The carrier concentration of the Si substrates was $5 \times 10^{14} \mathrm{~cm}^{-3}$. The electrical resistivity for $3 \mathrm{C}-\mathrm{SiC}(100)$ and $3 \mathrm{C}-\mathrm{SiC}(111)$ was found to be $0.14 \Omega \mathrm{cm}$ and 0.44 $\Omega \mathrm{cm}$ with corresponding hole mobility of $9 \mathrm{~cm}^{2} / \mathrm{Vs}$ and $1.88 \mathrm{~cm}^{2} / \mathrm{Vs}$, respectively.

Hall devices with a Greek cross shape were fabricated in different crystal orientations using conventional photolithography and dry etch processes (Fig. 2(a)) to investigate the stress-induced piezo-Hall effect. After fabrication of the device, the wafer was diced into strips with dimensions of $60 \mathrm{~mm} \times 9 \mathrm{~mm} \times 0.625 \mathrm{~mm}$ to apply stress by the bending beam method (supplementary document). A dedicated experimental setup capable of applying magnetic field and mechanical stress simultaneously was designed to measure the piezo-Hall effect as shown in Fig. 2(b). The method to numerically calculate the stress-induced into the $3 \mathrm{C}-\mathrm{SiC}$ layer on $\mathrm{Si}$ strip is reported elsewhere ${ }^{15,25}$. The applied stress induced in $\mathrm{SiC}$ layer was in the range of 0 to $264 \mathrm{MPa}$ (supplementary

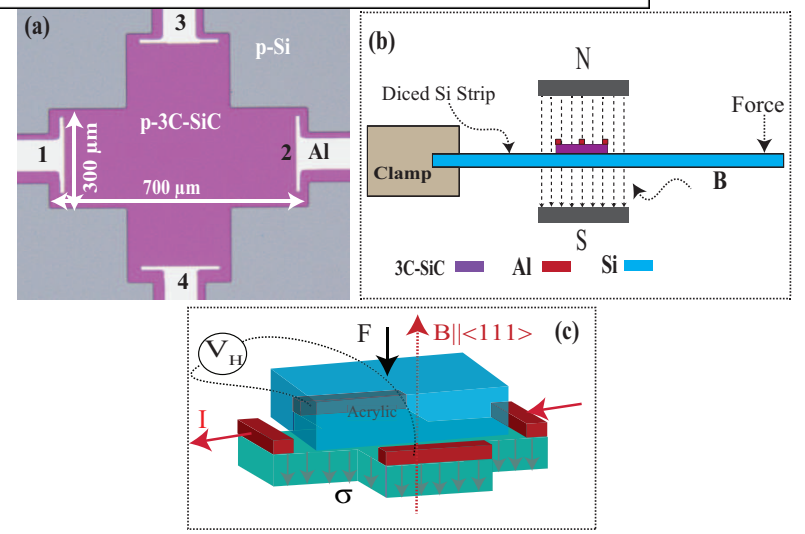

FIG. 2. (a) Microscopic image of the fabricated Hall device, (b) Experimental setup for simultaneous application of stress and magnetic field, (c) Experimental setup for applying stress along the thickness of thin film.

document). For the calculation of piezo-Hall coefficient $P_{11}$ the setup for applying stress along the thickness of thin film is shown in Fig. 2(c). The Ohmic contacts were confirmed by $I-V$ measurement and horizontal and vertical current leakages from $3 \mathrm{C}-\mathrm{SiC}(100)$ through to the $\mathrm{Si}$ substrate were meaured to be less than $0.5 \%$ of the total device current (supplementary document). A large valence band offset between p-Si and p-3C-SiC $(1.7 \mathrm{eV})$ prevents the leakage current through $\mathrm{SiC} / \mathrm{Si}$ junction ${ }^{16,17}$.

Figure 3 shows the variation of magnetic field sensitivity with applied stress for different orientations of the p-type 3C-SiC(100) Hall device. In the presence of a constant magnetic field $B$, when stress is applied to the device at constant input current, the Hall voltage across terminals 3 and 4 will change with the increase of applied stress, which is called piezo-Hall effect. The change in Hall voltage was used to calculate the change in magnetic field sensitivity of the Hall device according to the following relation:

$$
S=\left(V_{H} / I\right) / B=R_{H}(G / t) \quad\left[V A^{-1} T^{-1}\right]
$$

where $V_{H}$ is the Hall voltage observed at terminals 3 and $4, I$ is the current flowing through terminals 1 and 2 as shown in Fig. 2(a) and $B$ is the magnetic field. $R_{H}$ is the Hall coefficient and $G$ is the geometrical correction factor and $t$ is the thickness of the $3 \mathrm{C}-\mathrm{SiC}$ thin film. The sensitivity of Hall devices was measured for three different angles (i.e. $0^{\circ}, 45^{\circ}, 90^{\circ}$ ) between the current flow and the longitudinal stress. The stress was applied in two different crystal orientations (i.e [100] and [110]) for 3C-SiC(100) Hall devices (Fig. 3(a)). It can be observed from Fig. 3(a) that the relative change in magnetic field sensitivity has the equal magnitude in all the six orientations of the device in (100) crystal plane with a maximum error of $\pm 5 \%$. Figure 3(b) shows the change in magnetic field sensitivity of the Hall device in (111) crystal plane when stress was applied along the thickness of the film. The small variations in the magnetic field sensitivity were observed due to the difficulty of applying stress in this 
This manuscript was accepted by Appl. Phys. Lett. Click here to see the version of record.
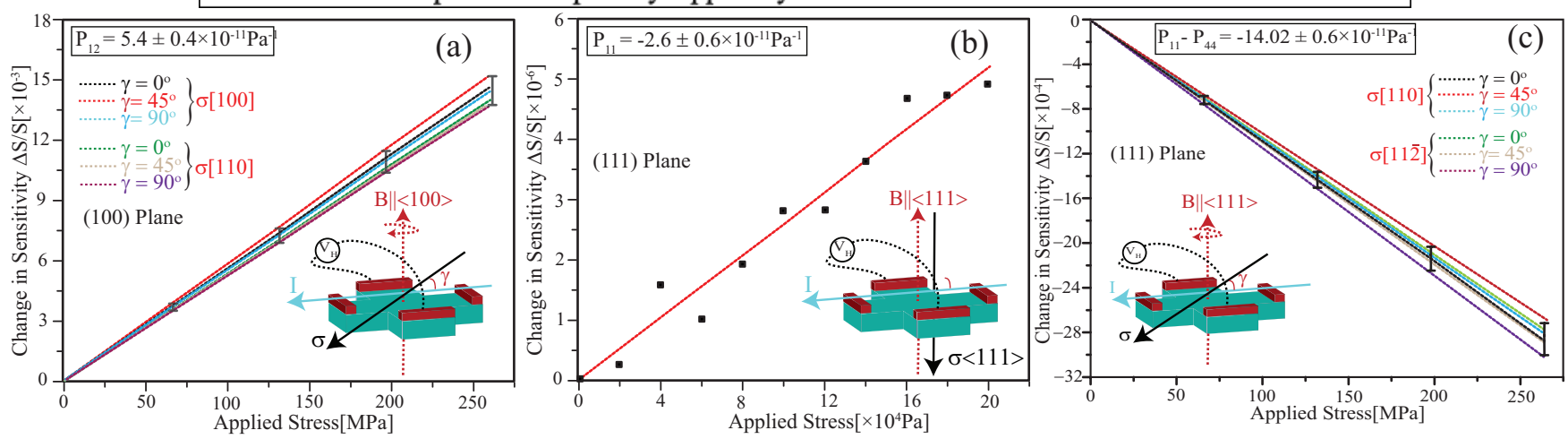

FIG. 3. Effect of applied stress on the magnetic field sensitivity of the fabricated Hall device for various angles of rotation within (100) crystal plane (a), for (111) crystal plane with stress along the thickness of device (b) and for (111) crystal plane with various angles of rotation. The insets show the directions of current, stress and magnetic field.

configuration and small amount of stress which can be induced by this method. A linear fitting was used to obtain the quantified values of magnetic field sensitivity. Figure 3(c) shows the variation of magnetic field sensitivity of $3 \mathrm{C}-\mathrm{SiC}(111)$ Hall devices in (111) crystal plane. The change in Hall voltage of these devices in (111) crystal plane was observed to be in opposite direction (i.e negative) as compared to the (100) crystal plane and it is almost same in all observed directions of stress and applied current with a maximum error of $\pm 5 \%$. Due to the crystal symmetry of $\mathrm{Si}$ to $\mathrm{SiC}$ the results are explained by the model presented for cubic Si by ${ }^{26}$ :

Let $\epsilon$ be a wafer plane of arbitrary direction. A unit vector $\vec{z}$ perpendicular to the wafer plane $\epsilon$ along with two orthogonal unit vectors $\vec{x}$ and $\vec{y}$ in plane $\epsilon$ are defined as:

$$
\left.\begin{array}{l}
\vec{z}=\sin \theta \cos \phi \vec{e}_{[100]}+\sin \theta \sin \phi \vec{e}_{[010]}+\cos \theta \vec{e}_{[001]} \\
\vec{x}=-\cos \theta \cos \phi \vec{e}_{[100]}-\cos \theta \sin \phi \vec{e}_{[010]}+\sin \theta \vec{e}_{[001]} \\
\vec{y}=\sin \phi \vec{e}_{[100]}-\cos \phi \vec{e}_{[010]}
\end{array}\right\}
$$

where the unit vectors $\vec{e}_{[100]}, \vec{e}_{[010]}$ and $\vec{e}_{[001]}$ point in the [100], [010] and [001] directions of the cubic SiC, respectively. A magnetic field is applied perpendicular to the wafer plane $\vec{B}=B \vec{z}$ and a current density $\vec{J}=J \vec{e}_{J}$ is expected to flow in wafer plane in arbitrary direction $\vec{e}_{J}=\cos \gamma \vec{x}+\sin \gamma \vec{y}$. The Hall electric field in plane $\epsilon$ perpendicular to $\vec{e}_{J}$ and $\vec{z}$ is given by $\vec{E}_{H}=E_{H} \vec{e}_{E}$, where $\vec{e}_{E}=\vec{z} \times \vec{e}_{J}$ (supplementary document). The Hall electric field can now be presented as:

$$
\overrightarrow{E_{H}}=\left[\begin{array}{lll}
\dot{R}_{11} & \dot{R}_{12} & \dot{R}_{13} \\
\dot{R}_{21} & \dot{R}_{22} & \dot{R}_{23} \\
\dot{R}_{31} & \dot{R}_{32} & \dot{R}_{33}
\end{array}\right]\left[\begin{array}{l}
\dot{B}_{1} \\
\dot{B}_{2} \\
\dot{B}_{3}
\end{array}\right] \times \vec{J}
$$

where $(i \hat{2} \dot{3})=(\vec{z} \vec{x} \vec{y})$ and $R_{i j}$ is the tensor of Hall coefficients. As the magnetic field is perpendicular to wafer, $\dot{B}_{1}=B, \dot{B}_{2}=0$ and $\dot{B}_{3}=0$. Therefore the Hall electric field is now given by (supplementary document):

$$
E_{H}=\dot{E}_{H}=\dot{\overrightarrow{E_{H}}} \cdot \dot{\overrightarrow{e_{E}}}=-B J \dot{R}_{11}
$$

It is evident from Eq. 4 that the angle $\gamma$ does not appear in the final equation of Hall electric field which shows that the piezo-Hall effect does not change when the device is rotated within the crystal plane which completely agrees with the experimental results as shown in Fig. 3. Therefore, unlike piezoresistive effect the Piezo-Hall effect is isotropic in any wafer plane. The Hall coefficient tensor $R_{11}^{\prime}=R_{0}(1+p h)$, where $R_{0}$ is the Hall coefficients without stress and $p h$ is contribution to Hall coefficient due to piezo-Hall effect and can be described as:

$$
p h=P_{11}^{\prime} \sigma_{1}^{\prime}+P_{12}^{\prime} \sigma_{2}^{\prime}+P_{13}^{\prime} \sigma_{3}^{\prime}+P_{14}^{\prime} \sigma_{4}^{\prime}+P_{15}^{\prime} \sigma_{5}^{\prime}+P_{16}^{\prime} \sigma_{6}^{\prime}
$$

where $\sigma_{1}$ is the normal stress orthogonal to wafer, $\sigma_{2}$ and $\sigma_{3}$ are in plane normal stresses, $\sigma_{4}$ is the in plane shear stress which vanishes at a distance of more than chip thickness form the edge of the chip, $\sigma_{5}$ and $\sigma_{6}$ are out of plane shear stresses which are normally zero. While, $P_{12}=P_{13}$ and $P_{14}=P_{15}=P_{16}=0$ due to the symmetry relations of the cubic crystal system $m 3 m$. Therefor, Eq. 5 reduces to:

$$
\left.\begin{array}{c}
p h=P_{11}^{\prime} \sigma_{1}+P_{12}^{\prime} \sigma_{2}^{\prime}+P_{13}^{\prime} \sigma_{3}^{\prime} \\
P_{11}^{\prime}=P_{11}-2 P_{a} \sin ^{2} \theta\left(\cos ^{2} \theta+\sin ^{2} \theta \sin ^{2} \phi \cos ^{2} \phi\right) \\
P_{12}^{\prime}=P_{12}+2 P_{a} \sin ^{2} \theta \cos ^{2} \theta\left(1-\sin ^{2} \phi \cos ^{2} \phi\right) \\
P_{13}^{\prime}=P_{12}+2 P_{a} \sin ^{2} \theta \sin ^{2} \phi \cos ^{2} \phi
\end{array}\right\}
$$

where $P_{a}=P_{11}-P_{12}-P_{44}$. In 3C-SiC(100) plane $\theta=$ $\phi=0^{\circ}$ when stress in [100] and $\theta=0^{\circ}, \phi=45^{\circ}$ when stress is in [110] direction. For p-type $3 \mathrm{C}-\mathrm{SiC}(111) \theta=$ $54.7^{\circ}$ and $\phi=45^{\circ}$. Incorporating these values in Eq.7 gives the final influence of stress to piezo-Hall effect for (100) and (111) plane in 3C-SiC as follows:

$$
\left.\begin{array}{l}
p h_{(100)}=\frac{\Delta S}{S}=P_{11} \sigma_{1}+P_{12}\left(\sigma_{2}+\sigma_{3}\right) \\
p h_{(111)}=\frac{\Delta S}{S}=\frac{P_{11}+2 P_{12}-P_{44}}{3} \sigma_{1}^{\prime}+\frac{P_{11}+2 P_{12}-P_{44}}{3}\left(\sigma_{2}+\sigma_{3}\right)
\end{array}\right\}
$$

When uni-axial stress $\sigma_{2}$ is applied then we have $\sigma_{1}=$ $\sigma_{3}=0$. Therefore, the corresponding piezo-Hall coefficient measured using Eq. 8 for p-type 3C-SiC(100) was 
This manuscript was accepted by Appl. Phys. Lett. Click here to see the version of record.

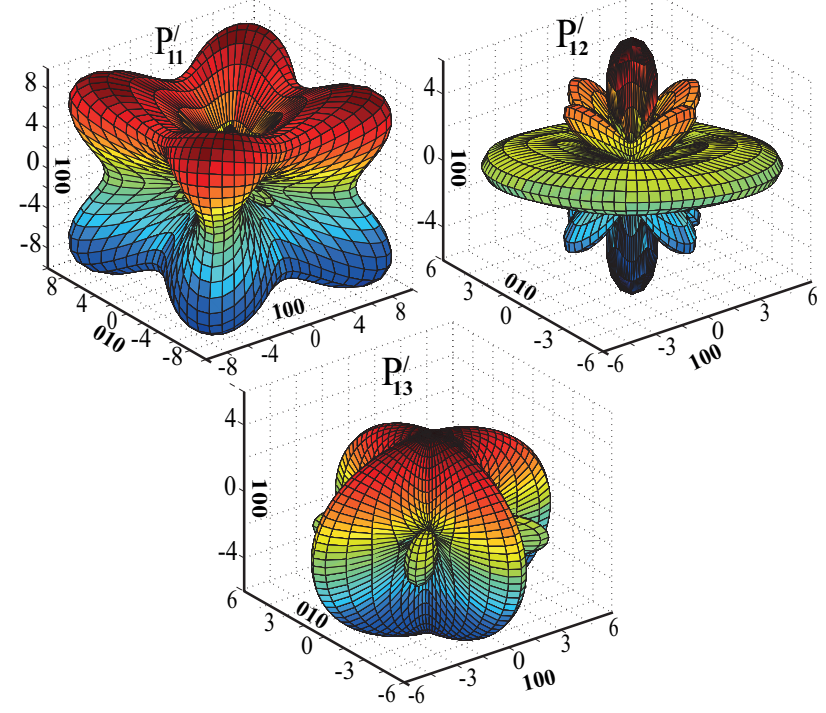

FIG. 4. Spherical plots of piezo-Hall coefficients for arbitrary crystal orientation in any wafer plane.

$P_{12}=(5.4 \pm 0.4) \times 10^{-11} \mathrm{~Pa}^{-1}$ and for p-type $3 \mathrm{C}-\mathrm{SiC}(111)$ the expression $P_{11}-P_{44}=(-14.2 \pm 0.6) \times 10^{-11} \mathrm{~Pa}^{-1}$ was measured (supplementary document). For the measurement of three independent piezo-Hall coefficients, three different configurations of magnetic field, applied stress and current are required. Two configurations were used in cantilever method and the third necessary configuration was obtained by applying stress along the thickness of the thin film in (111) crystal plane. From Eq. 8 for (111) plane with stress along [111] orientation the value of $P_{11}$ is determine to be $(-2.6 \pm 0.6) \times 10^{-11} \mathrm{~Pa}^{-1}$. Hence, the three independent piezo-Hall coefficients were obtained as; $P_{12}=(5.4 \pm 0.4) \times 10^{-11} \mathrm{~Pa}^{-1}, P_{11}=$ $(2.6 \pm 0.4) \times 10^{-11} \mathrm{~Pa}^{-1}$ and $P_{44}=(11.42 \pm 0.5) \times 10^{-11}$ $\mathrm{Pa}^{-1}$. Using the values of $P_{11}, P_{12}$ and $P_{44}$ the piezo-Hall coefficients for any arbitrary crystal orientation can be determined using Eq.7 and are given in Fig. 4 as spherical plots. It can be observed form the spherical plots of Fig. 4 that the piezo-Hall coefficient $P_{11}^{\prime}$ is minimum for $\{100\}$ planes and is maximum for $\{111\}$ planes. The piezo-Hall coefficient $P_{12}^{\prime}$ is minimum for $\{111\}$ planes and is maximum for $\{100\}$ planes. Similarly, the piezoHall coefficient $P_{13}^{\prime}$ is maximum for $\{111\}$ planes and minimum for $\{100\}$ planes.

In conclusion the piezo-Hall effect for single crystal p-type 3C-SiC has been investigated for different crystallographic orientations. It has been found that unlike piezoresistive effect, the piezo-Hall effect is isotropic i.e. it does not change with the rotation of the Hall device within the crystal plane. The piezo-Hall coefficients $P_{12}$, $P_{11}$ and $P_{44}$ for p-type $3 \mathrm{C}-\mathrm{SiC}$ have been determined which can then be used to determine the piezo-Hall coefficients in any crystallographic orientation. The $\{111\}$ planes of p-type 3C-SiC are more sensitive to stress than the $\{100\}$ planes. The results obtained in the study can be used to quantify the magnetic field drifts due to stress in the design of Hall effect sensors.

This work was performed in part at the Queensland node of the Australian National Fabrication Facility, a company established under the National Collaborative Research Infrastructure Strategy to provide nano and micro-fabrication facilities for Australia's researchers. This work has been partially supported by the Griffith University's New Researcher Grants.

The Supplementary Document provides detailed information about the fabrication process, current leakage, method of stress and mathematical derivations of the piezo-Hall coefficients.

${ }^{1}$ B. Halg, Journal of Applied Physics, 64, 276 (1988).

${ }^{2}$ A. Nathan and T. Manku, Applied Physics Letters, 62, 2947 (1993).

${ }^{3}$ R. G. Mani, K. von Klitzing, F. Jost, K. Marx, S. Lindenkreuz, and H. P. Trah, Applied Physics Letters, 67, 2223(1995).

${ }^{4}$ S. Huber and C. Schott and O. Paul, IEEE Sensors Journal, 13(8), 2890 (2013).

${ }^{5}$ Y. Liu, Z. L. Rang, A. K. Fung, C. Cai, P. P. Ruden, M. I. Nathan and H. Shtrikman, Applied Physics Letters, $79(27)$, 4586(2001).

${ }^{6}$ J. M. Cesaretti and W. P. Taylor and G. Monreal and O. Brand, IEEE Trans. Magn. 45(10), 4482 (2009).

${ }^{7} \mathrm{H}$. Husstedt and U. Ausserlechner and M. Kaltenbache, IEEE Sensors J., 11(11), 2993 (2011).

${ }^{8}$ R. Steiner and C. Maier and M. Mayer and S. Bellekom and H. Baltes, IEEE MEMS J., 8(4), 466 (2011).

${ }^{9}$ D. Manic and J. Petr and R.S. Popovic, Microelectronics Reliability, 41, 767 (1999).

${ }^{10} 1 \mathrm{M}$. Mehregany, C. A. Zorman, N. Rajan, and C. H. Wu, Proc. IEEE, 86(8), 1594 (1998).

${ }^{11}$ P. M. Sarro, Sens. Actuators A, Phys., 82(1-3), 210 (2000).

${ }^{12}$ S. Roy and C. Jacob and S. Basu, IEEE Trans. Electron Devices, 94, 298 (2003).

${ }^{13}$ F. La Via and M. Camarda and A. La Magna, Appl. Phys. Revs., 1, 031301 (2014).

${ }^{14}$ H.-P. Phan, D. V. Dao, K. Nakamura, S. Dimitrijev, and N.-T. Nguyen, J. Microelectromech. Syst., 24(6), 1663 (2015).

${ }^{15}$ H. P. Phan, D. V. Dao, P. Tanner, L. Wang, N. T. Nguyen, Y. Zhu, and S. Dimitrijev, Appl. Phys. Lett., 104(11), 111905 (2014).

${ }^{16}$ A. Qamar, P.Tanner, D. V. Dao, H. P. Phan and T. Dinh, Electron Device Letters, 35(12), 1293 (2014).

${ }^{17}$ A. Qamar, D.V. Dao, P. Tanner, H.P. Phan, T. Dinh, S. Dimitrijev, Applied Physics Express, 8(6), 061302 (2015).

${ }^{18}$ A. Qamar H.P. Phan, D.V. Dao, P. Tanner, T. Dinh, L. Wang and S. Dimitrijev, Electron Device Letters, 36(7), 708 (2015).

${ }^{19}$ A. Qamar, H.P. Phan, J. Han, P. Tanner, T. Dinh, L. Wang, D.V. Dao and S. Dimitrijev, J. Mater. Chem. C, 3, 8804 (2015).

${ }^{20}$ C.-M. Lin, Y.-Y. Chen, V. V. Felmetsger, D. G. Senesky, and A. P. Pisano, Adv. Mater. 24(20), 2722 (2012).

${ }^{21}$ C.-M. Lin, Y.-Y. Chen, V. V. Felmetsger, W.-C. Lien, T. Riekkinen, D. G. Senesky, and A. P. Pisano, J. Micromech. Microeng. 23, 025019 (2013).

${ }^{22}$ Afzaal Qamar, H-P Phan, Toan Dinh, Li Wang, Sima Dimitrijev and Dzung Viet Dao, RSC Advances, 6(37), 31191 (2016).

${ }^{23} \mathrm{Li}$ Wang, Sima Dimitrijev, J. Han P. Tanner, A. Iacopi and L. Hold, J. Cryst. Growth, 329(1), 67 (2011).

${ }^{24} \mathrm{Li}$ Wang, Alan Iacopi, Sima Dimitrijev, Glenn Walker, Alanna Fernandes, Leonie Hold, Jessica Chaia, Thin Solid Films, 564, 39 (2014).

${ }^{25}$ H. P. Phan, D. V. Dao, P. Tanner, N. T. Nguyen, J. S. Han, S. Dimitrijev, G. Walker, L. Wang and Y. Zhu, J. Mater. Chem. C, 2, 7176 (2014).

${ }^{26}$ A. Udo, IEEE Proc. Sensors, 3, 1149 (2004). 


\section{$\mathrm{N}$}

Diced Sị Strip

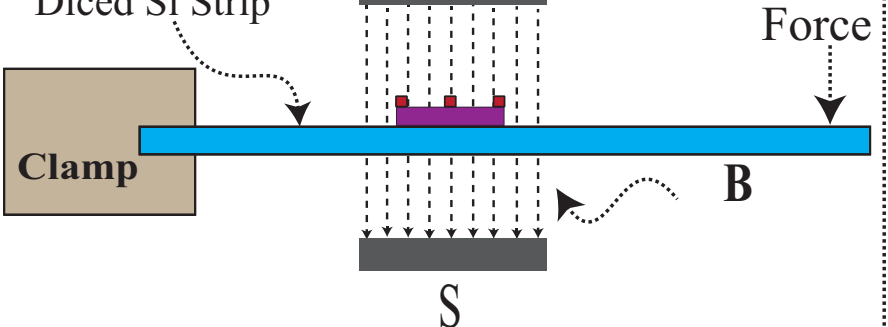

3C-SiC $\square \mathrm{Al}=\mathrm{Si}$

Force

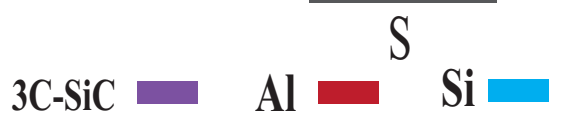

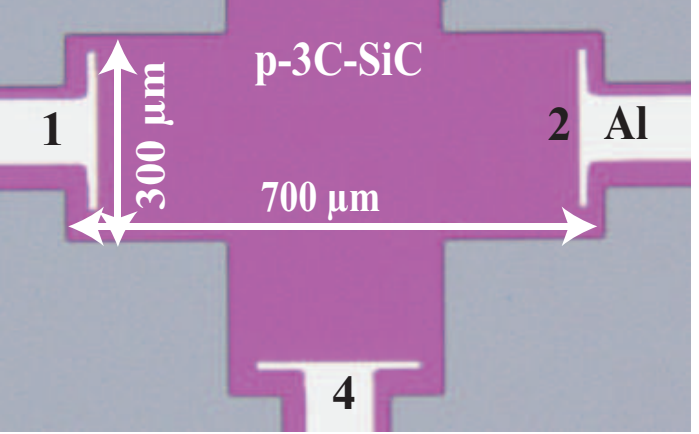

(b)

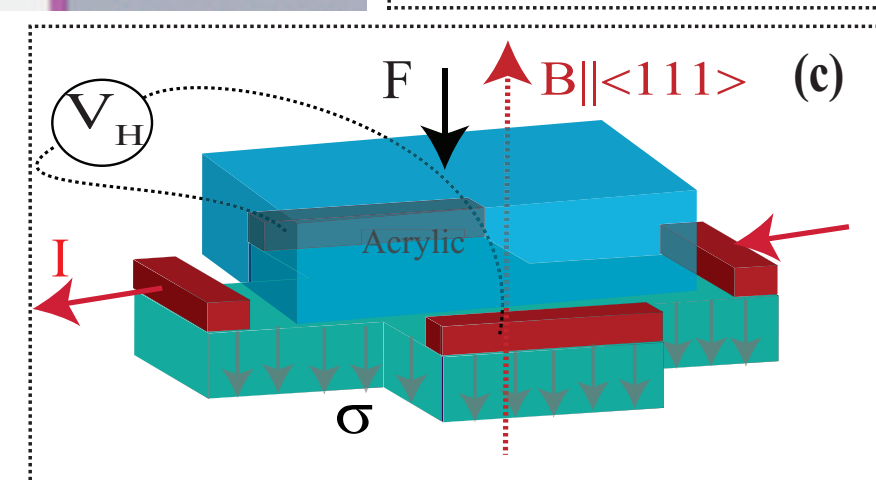

(c)

(a)

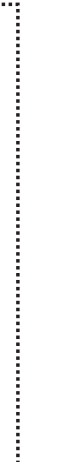




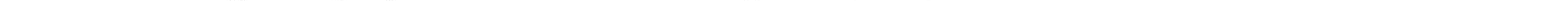




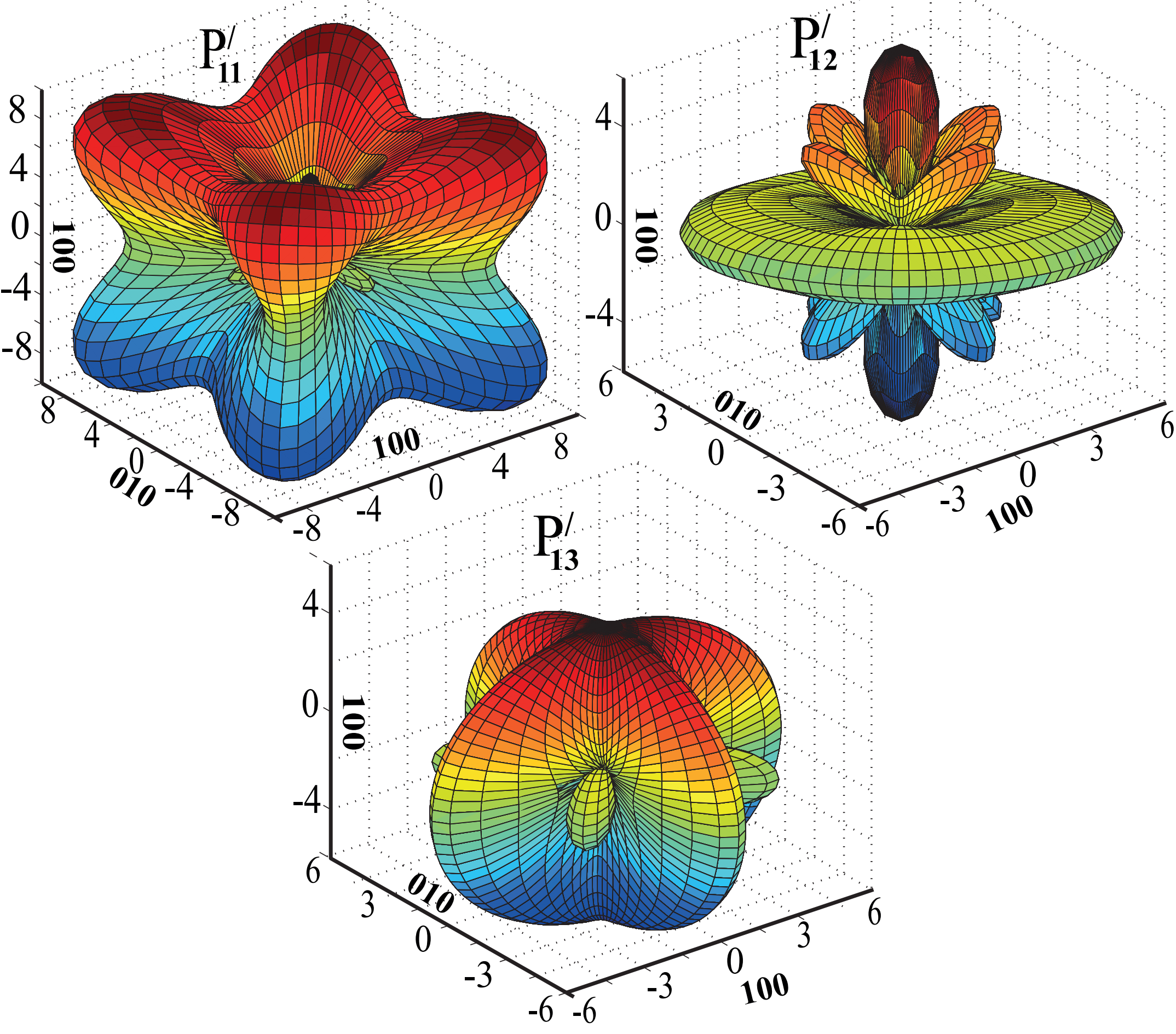

